\title{
Touchdown and related problems in electrostatic MEMS device equation
}

Nikos I. Kavallaris, Tosiya Miyasita and Takashi Suzuki

\begin{abstract}
We study the electrostatic MEMS-device equation, $u_{t}-\Delta u=$ $\frac{\lambda|x|^{\beta}}{(1-u)^{p}}$, with Dirichlet boundary condition. First, we describe the touchdown of non-stationary solution in accordance with the total set of stationary solutions. Then, we classify radially symmetric stationary solutions and their radial Morse indices. Finally, we show the Morse-Smale property for radially symmetric non-stationary solutions.
\end{abstract}

Mathematics Subject Classification (2000). Primary 35K55, 35J60; Secondary 74H35, 74G55, 74K15.

Keywords. Electrostatic MEMS, touchdown-quenching, Morse idex.

\section{Introduction}

The purpose of the present paper is to study the global-in-time behaviour of the solution to the parabolic problem

$$
\begin{aligned}
& u_{t}-\Delta u=\frac{\lambda f(x)}{(1-u)^{p}}, \quad 0 \leq u<1 \quad \text { in } \Omega \times(0, T) \\
& u=0 \quad \text { on } \partial \Omega \times(0, T) \\
& \left.u\right|_{t=0}=u_{0}(x) \quad \text { in } \Omega,
\end{aligned}
$$

where $\lambda>0$ is a constant, $p>1, f(x) \geq 0, f(x) \not \equiv 0$ is a continuous function, $\Omega \subset \mathbb{R}^{n}$ is a bounded domain with smooth boundary $\partial \Omega$, and $u_{0}=u_{0}(x) \in[0,1)$ is a continuous function. This equation models the dynamic deflection of an elastic mebrane inside a micro-electro mechanical system (MEMS). This kind of systems combine electronics with various types of micro-size mechanical devices and could be found in accelerometers for airbag deployment in automobiles, in ink jet printer heads, in optical switches, in chemical sensors and so on, for more details see [21] and the references therein.

On leave to Department of Statistics and Actuarial Science, University of the Aegean, current email address:nkaval@aegean.gr 
The structure of the set of stationary solutions has been studied for $p=2$ $([8,11])$. We obtain, similarly, an upper bound of $\lambda$ for the existence of the solution denoted by $\bar{\lambda}>0$ for general $p>1$. Thus, if $0<\lambda<\bar{\lambda}$ and $\lambda>\bar{\lambda}$, then $\mathcal{C}^{\lambda} \neq \emptyset$ and $\mathcal{C}^{\lambda}=\emptyset$, respectively, where

$$
\mathcal{C}^{\lambda}=\left\{u \in C^{2}(\Omega) \cap C_{0}(\bar{\Omega}) \mid u \text { solves }(1.1)\right\} .
$$

Also, if $\mathcal{C}^{\lambda} \neq \emptyset$ there is a unique minimal element, denoted by $u_{\lambda}$.

The global-in-time behaviour of the non-stationary solution has been studied also for $p=2([9])$. More precisely, for $\lambda<\bar{\lambda}$ and $\lambda>\bar{\lambda}$, there is a solution to (1.1) converging to $u_{\lambda}$ uniformly and any solution must touchdown in finite time, i.e.,

$$
T<+\infty, \quad \lim _{t \uparrow T}\|u(\cdot, t)\|_{\infty}=1,
$$

respectively. Here and henceforth, $T>0$ denotes the existence time of the solution. We can refine these results on the global-in-time behaviour of the solution even for general $p>1$.

Theorem 1.1. We have the following.

1. If $\lambda>\bar{\lambda}$, then (1.2) occurs.

2. If there is $u^{*} \in \mathcal{C}^{\lambda} \backslash\left\{u_{\lambda}\right\}$ and $u_{0} \leq u^{*}, u_{0} \neq \equiv u^{*}$ or $\mathcal{C}^{\lambda}=\left\{u_{\lambda}\right\}$ and $u_{0} \leq u_{\lambda}$, then $T=+\infty$ and $u(\cdot, t) \rightarrow u_{\lambda}$ uniformly as $t \uparrow+\infty$.

3. If there is $u^{*} \in \mathcal{C}^{\lambda} \backslash\left\{u_{\lambda}\right\}, u_{0} \geq u^{*}, u_{0} \not \equiv u^{*}$, then (1.2) occurs.

Next, we take the case of

$$
\Omega=B \equiv\left\{x \in \mathbb{R}^{n}|| x \mid<1\right\}, \quad f(x)=|x|^{\beta}, \quad \beta \geq 0,
$$

and prove existence of non-minimal radially symmetric stationary solutions. To state the results, we set

$$
\begin{aligned}
\mathcal{C}_{r}^{\lambda} & =\left\{u \in C^{2}(\Omega) \cap C_{0}(\bar{\Omega}) \mid u=u(|x|) \text { solves }(1.1)\right\} \\
\mathcal{C}_{r} & =\bigcup_{\lambda>0}\{\lambda\} \times \mathcal{C}_{r}^{\lambda}
\end{aligned}
$$

and

$$
\begin{aligned}
& \bar{\beta}=\frac{p\{2(p-1)-(p+1) n\}+(n-2)(p+1) \sqrt{p(p+1)}}{2 p} \\
& \bar{p}=\frac{-\left(n^{2}-8 n+4\right)-8 \sqrt{n-1}}{(n-2)(n-10)}
\end{aligned}
$$


where $\bar{\beta}$ and $\bar{p}$ are the maximum solutions of the equations $f(p, n, \beta)=0$ and $f(p, n, 0)=0$ respectively, for $f(p, n, \beta)=(p+1)^{2} n^{2}-4(p+1)(\beta p+3 p+1) n-$ $4\left\{\beta^{2} p+2 p \beta(1-p)-\left(5 p^{2}+2 p+1\right)\right\}$. Note that $\bar{\beta}, \bar{p}$ are defined for $n \geq 7$ and $n=7,8,9$ respectively.

Theorem 1.2. If $n \geq 2$, then $\mathcal{C}_{r}$ is homeomorphic to $\mathbb{R}$ and has end points $(0,0)$ and $\left(\lambda^{*}, 1-|x|^{\frac{\beta+2}{p+1}}\right)$, where

$$
\lambda^{*}=\frac{(\beta+2)\{\beta+2+(n-2)(p+1)\}}{(p+1)^{2}} .
$$

In the cases of

$$
\begin{array}{ll}
n \in[2,6], & p>1, \beta>0, \\
n \in[7,9], & p>\bar{p}, \beta>0, \quad \text { or } \quad p \in(1, \bar{p}), \beta>\bar{\beta}, \\
n \geq 10, & p>1, \beta>\bar{\beta},
\end{array}
$$

whenever $\bar{\beta}>0, \mathcal{C}_{r}$ bends infinitely many times with respect to $\lambda$ around $\lambda^{*}$, while at most a finite number of bendings occur to $\mathcal{C}_{r}$ when

$$
\begin{array}{ll}
n \in[7,9], & p \in(1, \bar{p}], \beta \in(0, \bar{\beta}], \\
n \geq 10, & p>1, \beta \in(0, \bar{\beta}] .
\end{array}
$$

The case $p=2$ has also been studied on $\mathcal{C}=\bigcup_{\lambda>0}\{\lambda\} \times \mathcal{C}^{\lambda}$. First, the above profile of $\mathcal{C}_{r}$ is observed numerically [8]. Next, some estimates on $\bar{\lambda}$ are given for nonradially symmetric case $[8,9]$. Finally, existence of the non-minimal non-radially symmetric stationary solution is proven by the variational method [4].

Given $u \in \mathcal{C}^{\lambda}$, the linearized eigenvalue problem is defined as follows:

$$
-\Delta \phi-\frac{p \lambda f(x)}{(1-u)^{p+1}} \phi=\mu \phi \text { in } \Omega, \quad \phi=0 \text { on } \partial \Omega .
$$

Then, the number of its negative eigenvalues, denoted by $i=i(\lambda, u)$, is called Morse index. In case (1.3) the number of negative eigenvalues corresponding to radially symmetric eigenfunctions, denoted by $i_{R}=i_{R}(\lambda, u)$, is called radial Morse index.

Theorem 1.3. Under the assumptions of Theorem 1.2, $i_{R}=i_{R}(\lambda, u)$ increases one by one at each bending point.

A general theorem guarantees $i(u, \lambda)=i_{R}(u, \lambda)$ for

$$
-\Delta u=\lambda f(r, u) \geq 0 \text { in } B, \quad u=0 \text { on } \partial B,
$$


if $f_{r} \leq 0$, where $r=|x|$, see [16]. This is not the case of (1.3) with $\beta>0$. In fact, general theorems of radial symmetry of the solution to the stationary problem are not valid for $\beta>0([10,20])$. There may be non-radial bifurcation and $i(\lambda, u)=i_{R}(\lambda, u)$ would not be always valid.

This paper is composed of five sections. Theorems 1.1, 1.2, and 1.3 are proven in sections 2,3 , and 4 , respectively. In the final section, $\S 5$, we confirm the role of radial Morse indices in the formation of connecting orbits.

\section{2. $\quad$ Proof of Theorem 1.1}

The original proof for $p=2$ to the first case of this theorem is quite technical [9], it provides with some bounds of the blow-up time though. Here we give a simpler proof by applying Kaplan's method adopted by [2]. The proof of the second case is obtained by using classical techniques like maximum-principle or dynamical-systems aguments, [7, 9], and we omit it. The final case is obtained by the method of [15].

Before proceeding to these cases, we recall that Kaplan's method guarantees touchdown for any $\lambda>0$ if the initial value is close to 1 and $f>0$ on $\bar{\Omega}$ (see also [3]). In fact, let $\mu_{1}>0$ be the principal eigenvalue of $-\Delta$, and take the solution to

$$
-\Delta \varphi_{1}=\mu_{1} \varphi_{1}, \quad \varphi_{1}>0 \quad \text { in } \Omega, \quad \varphi_{1}=0 \quad \text { on } \partial \Omega,
$$

normalized by $\int_{\Omega} \varphi_{1}=1$. Then, using $m=\inf _{\Omega} f>0$ and $A=\int_{\Omega} u \varphi_{1} d x$, we obtain via Jensen's inequality

$$
\frac{d A}{d t} \geq-\mu_{1}+\lambda m \int_{\Omega}(1-u)^{-p} \varphi_{1} d x \geq-\mu_{1} A+\lambda m(1-A)^{-p} .
$$

Let $H(s)=-\mu_{1} s+\lambda m(1-s)^{-p}, s \in(0,1)$. Then there exists $a \in(0,1)$ such that $H(s)>0$ in $(a, 1)$. Hence if we choose $u_{0}$ close enough to 1 so that $A(0)>a$, then $\frac{d A}{d t}>0$ for all $t$ when $u$ is global. But, then we have

$$
t=\int_{0}^{t} d \sigma \leq \int_{A(0)}^{A(t)} \frac{d s}{H(s)} \leq \int_{A(0)}^{1} \frac{d s}{H(s)}<+\infty
$$

which is a contradiction. Kaplan's method could be also used to prove touchdown for big enough $\lambda$, see [11].

Now, we show the first case of Theorem 1.1, i.e., $\lambda>\bar{\lambda}$ implies (1.2). It suffices to assume $u_{0}=0$ for this purpose. Then, it holds that

$$
\begin{aligned}
& v_{t}-\Delta v=\frac{p \lambda f(x)}{(1-u)^{p+1}} v \quad \text { in } \Omega \times(0, T) \\
& v=0 \quad \text { on } \partial \Omega \times(0, T) \\
& \left.v\right|_{t=0}=\lambda f(x) \geq 0 \quad \text { in } \Omega,
\end{aligned}
$$


for $v=u_{t}$ and hence $u_{t} \geq 0$. In particular, $\lim _{t \uparrow T}\|u(\cdot, t)\|_{\infty}=1$ follows from $T<+\infty$, because $\mathcal{C}^{\lambda}=\emptyset$.

We assume $T=+\infty$ and multiply the first equation of (1.1) by $\varphi_{1}$ :

$$
\int_{t}^{t+1} \int_{\Omega} u_{t} \varphi_{1} d x d s=\int_{t}^{t+1} \int_{\Omega}(\Delta u) \varphi_{1} d x d s+\lambda \int_{t}^{t+1} \int_{\Omega} \frac{f(x) \varphi_{1}}{(1-u)^{p}} d x d s .
$$

Then, it holds that

$$
\begin{aligned}
& \lambda \int_{t}^{t+1} \int_{\Omega} \frac{f(x) \varphi_{1}}{(1-u)^{p}} d x d s \\
& \quad=\int_{\Omega} u(\cdot, t+1) \varphi_{1} d x-\int_{\Omega} u(\cdot, t) \varphi_{1} d x+\mu_{1} \int_{t}^{t+1} \int_{\Omega} u \varphi_{1} d x d s \\
& \quad \leq\|u(\cdot, t+1)\|_{\infty}+\mu_{1}\|u(\cdot, t)\|_{\infty} \leq 1+\mu_{1}
\end{aligned}
$$

by $0 \leq u(x, t)<1$ in $\Omega \times(0,+\infty)$, and therefore,

$$
\lambda \int_{\Omega} \frac{f(x) \varphi_{1}}{(1-u(x, t))^{p}} d x \leq 1+\mu_{1}
$$

by $u_{t} \geq 0$. This implies

$$
\sup _{t \geq 0} \int_{\Omega} \frac{f(x) \varphi_{1}}{(1-u(\cdot, t))^{p}} d x \leq \frac{1+\mu_{1}}{\lambda}<+\infty .
$$

From the monotone convergence theorem, and

$$
\|u(\cdot, t)\|_{1}=\int_{\Omega}|u(x, t)| d x \leq|\Omega|<+\infty,
$$

it follows that $u(\cdot, t)$ has a limit $w$ in $L^{1}(\Omega)$ as $t \rightarrow \infty$, while $K(x, t)=\frac{f(x)}{(1-u)^{p}}$ converges to $\tilde{K}(x)=\frac{f(x)}{(1-w)^{p}}$ in $L^{1}(\Omega, \delta(x) d x)$ as $t \uparrow+\infty$, where $\delta(x)=\operatorname{dist}(x, \partial \Omega)$. Testing (1.1) by $\varphi \in C^{2}(\bar{\Omega})$ with $\left.\varphi\right|_{\partial \Omega}=0$, we obtain

$$
\left[\int_{\Omega} u \varphi d x\right]_{t}^{t+1}=\int_{t}^{t+1} \int_{\Omega} u \Delta \varphi d x d s+\lambda \int_{t}^{t+1} \int_{\Omega} \frac{f(x) \varphi}{(1-u)^{p}} d x d s .
$$

Letting $t \rightarrow \infty$ we derive

$$
\int_{\Omega} w(-\Delta \varphi) d x=\int_{\Omega} \frac{\lambda f(x) \varphi}{(1-w)^{p}} d x .
$$


This means that $w$ is a weak solution to

$$
-\Delta w=\frac{\lambda f(x)}{(1-w)^{p}} \quad \text { in } \Omega, \quad w=0 \quad \text { on } \partial \Omega,
$$

and we obtain a contradiction by the following lemma, see also [2, 9].

Lemma 2.1. Problem (2.2) has no weak solutions for $\lambda>\bar{\lambda}$.

Proof. We define

$$
\begin{gathered}
g(w)=\frac{1}{(1-w)^{p}}, \quad h(w)=\int_{0}^{w} \frac{d s}{g(s)}=\int_{0}^{w}(1-s)^{p} d s \\
g_{\varepsilon}(w)=(1-\varepsilon) g(w), \quad h_{\varepsilon}(w)=\int_{0}^{w} \frac{d s}{g_{\varepsilon}(s)}=\frac{1}{1-\varepsilon} h(w)
\end{gathered}
$$

and put $\Phi_{\varepsilon}(w)=h_{\varepsilon}^{-1}(h(w))$, where $\varepsilon>0$. The following properties of this mapping $w \in[0,1) \mapsto \Phi_{\varepsilon}(w)$ are obtained by $g^{\prime \prime}>0$ :

$$
\begin{aligned}
\Phi_{\varepsilon}(0) & =0, \quad 0 \leq \Phi_{\varepsilon}(w)<w, \\
\Phi_{\varepsilon}^{\prime}(w) & =(1-\varepsilon) g\left(\Phi_{\varepsilon}(w)\right) g(w)^{-1}>0, \\
\Phi_{\varepsilon}^{\prime \prime}(w) & =(1-\varepsilon) g\left(\Phi_{\varepsilon}(w)\right)\left((1-\varepsilon) g^{\prime}\left(\Phi_{\varepsilon}(w)\right)-g^{\prime}(w)\right) g(w)^{-2} \\
& <(1-\varepsilon) g\left(\Phi_{\varepsilon}(w)\right)\left((1-\varepsilon) g^{\prime}(w)-g^{\prime}(w)\right) g(w)^{-2} \leq 0 .
\end{aligned}
$$

Given a weak solution $w=w(x) \in[0,1]$ to $(2.2)$ satisfying $\frac{f(x)}{(1-w)^{p}} \in$ $L^{1}(\Omega, \delta(x) d x)$, we define $w_{\varepsilon}=\Phi_{\varepsilon}(w)$. This means $h\left(w_{\varepsilon}\right)=(1-\varepsilon) h(w)$ and there is $\delta>0$ such that $0 \leq w_{\varepsilon} \leq 1-\delta$. It also holds by the concavity of $\Phi_{\epsilon}$ that

$$
-\Delta w_{\varepsilon} \geq \Phi_{\varepsilon}^{\prime}(w) \frac{\lambda f(x)}{(1-w)^{p}} \quad \text { in } \Omega, \quad w_{\varepsilon}=0 \quad \text { on } \partial \Omega
$$

in weak sense, i.e.,

$$
\begin{aligned}
-\int_{\Omega} w_{\varepsilon} \Delta J d x & \geq \lambda \int_{\Omega} \Phi_{\varepsilon}^{\prime}(w) \frac{f(x)}{(1-w)^{p}} J d x \\
& =\lambda(1-\varepsilon) \int_{\Omega} \frac{f(x) J}{\left(1-w_{\varepsilon}\right)^{p}} d x
\end{aligned}
$$

for any $J \in C^{2}(\bar{\Omega})$ satisfying $J \geq 0$ and $\left.J\right|_{\partial \Omega}=0$. Thus, $w_{\varepsilon}$ is a weak supersolution to (2.2) and therefore, the iteration sequence $\left\{v_{k}\right\}_{k=0}^{\infty}$ defined by

$$
-\Delta v_{k+1}=\frac{\lambda(1-\varepsilon) f(x)}{\left(1-v_{k}\right)^{p}} \quad \text { in } \Omega, \quad v_{k+1}=0 \quad \text { on } \partial \Omega
$$


with $v_{0}=w_{\varepsilon}$ is monotone decreasing. It also satisfies $v_{k} \geq 0$ and converges uniformly to a solution to $(2.2)$ for $\lambda(1-\varepsilon)$ by Dini's theorem. This implies $\mathcal{C}^{\lambda(1-\varepsilon)} \neq \emptyset$, a contradiction by the definition of $\bar{\lambda}$ since $\varepsilon>0$ is arbitrary.

The third case of Theorem 1.1 is a consequence of the following theorem.

Theorem 2.2. If there is a pair of super and sub-solutions to (2.2), $\bar{v}, \underline{v} \in$ $C^{2}(\Omega) \cap C_{0}(\bar{\Omega})$ satisfying $0 \leq \bar{v} \leq \underline{v}<1$ and $\bar{v} \not \equiv \underline{v}$, and if the initial value satisfies $\underline{v} \leq u_{0}<1$ and $\underline{v} \not \equiv u_{0}$ in (1.1), then it holds that $T<+\infty$ and $\lim _{t \uparrow T}\|u(\cdot, t)\|_{\infty}=1$.

Proof. Since $\bar{v}$ is a super-solution, the iteration sequence $\left\{v_{k}\right\}_{k=0}^{\infty}$ defined by (2.3) with $v_{0}=\bar{v}(x) \in[0,1)$ is monotone decreasing. It satisfies $v_{k} \geq 0$, and converges uniformly to a solution to (2.2) by Dini's theorem. Thus, we can assume that $\bar{v}$ is a classical solution to $(2.2)$.

Let $u_{1}$ and $u_{2}$ be the local in time solutions to (1.1) with $u_{0}=\underline{v}$ and $u_{0}=\bar{v}$, respectively. Then, by the strong maximum principle and the Hopf's lemma we have $u\left(\cdot, t_{0}\right) \gg u_{1}\left(\cdot, t_{0}\right) \gg u_{2}\left(\cdot, t_{0}\right)$ for $0<t_{0} \ll 1$, i.e.,

$$
\begin{aligned}
& u\left(\cdot, t_{0}\right)>u_{1}\left(\cdot, t_{0}\right)>u_{2}\left(\cdot, t_{0}\right) \quad \text { in } \Omega, \\
& \frac{\partial u}{\partial \nu}\left(\cdot, t_{0}\right)<\frac{\partial u_{1}}{\partial \nu}\left(\cdot, t_{0}\right)<\frac{\partial u_{2}}{\partial \nu}\left(\cdot, t_{0}\right) \quad \text { on } \partial \Omega,
\end{aligned}
$$

where $\nu$ denotes the outer unit normal vector. Since $\underline{v}$ is a sub-solution of (2.2) we obtain, by the maximum principle, that $u_{1}$ is increasing in time, i.e., $u_{1 t}>0$, and hence $u_{1}\left(\cdot, t_{0}\right)$ is a strict sub-solution of problem (2.2). On the other hand, we obtain $u_{2}(\cdot, t)=\bar{v}$ because $\bar{v}$ is supposed to be a stationary solution. Therefore, we may assume that $\underline{v}$ and $\bar{v}$ are a strict sub-solution and a solution of (2.2), respectively, and that $u_{0}, \underline{v}, \bar{v}$ are $C^{2}$ functions on $\bar{\Omega}$ satisfying

$$
u_{0} \gg \underline{v} \gg \bar{v} \text {. }
$$

In this case, we can take a constant $\theta>1$ such that

$$
u_{0} \gg \theta \underline{v}+(1-\theta) \bar{v}>\bar{v},
$$

and therefore, we can assume $u_{0}=\theta \underline{v}+(1-\theta) \bar{v}$ by the comparison theorem. This $u_{0}=u_{0}(x) \in[0,1)$ is a strict sub-solution because $g(u)=(1-u)^{-p}$ is convex. In fact,

$$
\begin{aligned}
-\Delta u_{0} & =-\theta \Delta \underline{v}-(1-\theta) \Delta \bar{v}<\frac{\lambda \theta f(x)}{(1-\underline{v})^{p}}+\frac{\lambda(1-\theta) f(x)}{(1-\bar{v})^{p}} \\
& =\lambda f(x)[\theta g(\underline{v})+(1-\theta) g(\bar{v})]<\lambda f(x) g\left(u_{0}\right) .
\end{aligned}
$$

Then, $u(x, t)$ is strict increasing in $t$ for each $x \in \Omega$ and so we have a measurable function $v(x)$ such that

$$
\lim _{t \uparrow+\infty} u(x, t)=v(x) \in(\bar{v}(x), 1] \quad \text { for } x \in \Omega
$$


under the assumption $T=+\infty$. Then, similarly to the first case, it holds that

$$
\int_{\Omega} \frac{f(x) \varphi_{1}}{(1-v)^{p}} d x \leq \frac{1+\mu_{1}}{\lambda}<+\infty
$$

Using Duhamel's principle, we can write the solution of problem (1.1) in the form

$$
u(x, t)=\int_{\Omega} U(x, y ; t) u_{0}(y) d y+\int_{0}^{t} d s \int_{\Omega} U(x, y ; s) \frac{\lambda f(y)}{(1-u(y, t-s))^{p}} d y
$$

where $U(x, y ; t)>0$ is the fundamental solution of the heat operator. The second term of the right-hand side is written as

$$
\int_{0}^{\infty} \int_{\Omega} U(x, y ; s) \frac{\lambda f(y)}{(1-u(y, t-s))^{p}} \chi_{[0, t]}(s) d y d s
$$

where the monotone convergence theorem is applicable by (2.4). Since

$$
\lim _{t \uparrow+\infty} \int_{\Omega} U(x, y ; t) u_{0}(y) d y=0
$$

it holds that

$$
v(x)=\int_{0}^{\infty} d s \int_{\Omega} U(x, y ; s) \frac{\lambda f(y)}{(1-v(y))^{p}} d y
$$

for each $x \in \Omega$. Here, using Fubini's theorem, we obtain

$$
v(x)=\lambda \int_{\Omega} G(x, y) \frac{f(y)}{(1-v(y))^{p}} d y
$$

by $\int_{0}^{\infty} U(x, y ; t) d t=G(x, y)$, where $G(x, y)$ denotes the Green's function of $-\Delta$. This implies that $v=v(x) \in(\bar{v}(x), 1]$ is a weak solution to $(2.2)$.

We have

$$
\bar{v} \ll u_{0} \ll u\left(\cdot, t_{0}\right) \ll v
$$

and therefore, there is $\beta \in(0,1)$ such that

$$
u_{0} \leq \beta v+(1-\beta) \bar{v} \equiv z .
$$


Here, $\bar{v}$ is a classical solution to $(2.2)$, while $v=v(x) \in[\bar{v}(x), 1]$ is a weak solution to (2.2) satisfying $\frac{f(x)}{(1-v)^{p}} \in L^{1}(\Omega, \delta(x) d x)$. Then, we can define the minimal solution, denoted by $\tilde{u}$ to (1.1) with the initial value $z$ by the iteration scheme

$$
\begin{aligned}
\tilde{u}_{k+1}(x, t) & =\int_{\Omega} U(x, y ; t) z(y) d y+\int_{0}^{t} \int_{\Omega} \frac{U(x, y ; s) \lambda f(y)}{\left(1-\tilde{u}_{k}(y, t-s)\right)^{p}} d y d s \\
\tilde{u}_{0}(x, t) & =\int_{\Omega} U(x, y ; t) z(y) d y .
\end{aligned}
$$

In fact, this $\left\{\tilde{u}_{k}\right\}_{k=0}^{\infty}$ is monotone increasing in $k$ and satisfies

$$
\tilde{u}_{k}(x, t) \leq z(x)
$$

because $g(u)=(1-u)^{-p}$ is convex, and then $\tilde{u}(x, t)$ is defined by

$$
\lim _{k \rightarrow \infty} \tilde{u}_{k}(x, t)=\tilde{u}(x, t) .
$$

If $\left\{u_{k}\right\}_{k=0}^{\infty}$ is defined by

$$
\begin{aligned}
u_{k+1}(x, t) & =\int_{\Omega} U(x, y ; t) u_{0}(y) d y+\int_{0}^{t} \int_{\Omega} \frac{U(x, y ; s) \lambda f(y)}{\left(1-u_{k}(y, t-s)\right)^{p}} d y d s \\
u_{0}(x, t) & =\int_{\Omega} U(x, y ; t) u_{0}(y) d y,
\end{aligned}
$$

then the monotonicity of $g(s)$ implies that $u_{k} \leq \tilde{u}_{k}$ for each $k$ and hence $\lim _{k \rightarrow \infty} u_{k}(x, t)=$ $u(x, t)$. This implies

$$
v \leq \beta v+(1-\beta) \bar{v} \quad \text { in } \Omega
$$

by letting $k \rightarrow \infty$ and then $t \uparrow+\infty$. Then, it follows that $v \leq \bar{v}$, a contradiction to $(2.4)$.

In the case that problem (2.2) has only a weak solution $w^{*}$, i.e. $\left\|w^{*}\right\|_{\infty}=1$ and $w^{*}$ satisfies (2.2) in the sence of (2.1), we can prove that an infinite-time touchdown occurs for the solution of problem (1.1). More precisely we have the following.

Theorem 2.3. If proplem (2.2) has only a weak solution $w^{*}$, then for $u_{0}(x)<$ $w^{*}(x)$ there exists a unique gloabal solution $u^{*}$ of (1.1) such that $u^{*}(x, t) \leq w^{*}(x)$ in $\Omega$ for all $0<t<\infty$, while $u^{*}(x, t)$ converges pointwise to $w^{*}$ as $t \rightarrow \infty$. In particular, $\lim _{t \uparrow \infty}\left\|u^{*}(\cdot, t)\right\|_{\infty}=1$.

The proof of Theorem 2.3 follows the same steps with the proof of Theorem 2.5, for $p=2$, in [9] and uses some arguments introduced in [2]. 
An natural question arises, from the above theorem, if the rate of convergence of $u^{*}$ towards the singular steady state $w^{*}$ could be determined. Since this is a very interesting question and the answer requires a delicate analysis it will be addressed in a forthcoming paper.

The problem of the characterization of the set of the touchdown points, for the case $p=2$, has been first tackled in [9], while some more refined results were given in [12]. In particular, in [12], it is proven, for problem

$$
\begin{aligned}
& v_{t}-\Delta v=-\frac{\lambda f(x)}{v^{2}}, \quad 0 \leq v<1 \quad \text { in } \Omega \times(0, T) \\
& v=1 \quad \text { on } \partial \Omega \times(0, T) \\
& \left.v\right|_{t=0}=1 \quad \text { in } \Omega,
\end{aligned}
$$

that the finite-time touchdown set $Q:=\left\{x \in \Omega: v(x, T)=0, T=T_{\max }<\infty\right\} \subset$ $A:=\{x \in \Omega: f(x) \neq 0\}$ under the hypothesis that $Q$ is a compact set, which is the case when $\Omega$ is convex and $f$ satisfies some extra condition. Under the same hypothesis some upper and lower estimates of the touchdown profile are given as well as some refined touchdown profiles are provided, in the radial symmetric case, coming out by classical arguments like similarity-variables and central-manifolds techniques. In the case that $\Omega$ is not convex the question of determining the touchdown rate is still open, and probably some scaling arguments, which have been intoduced for general domains, could work in this case. Now, for the radial symmetric case, it is proven in [9], by using ideas from [5], that the origin $r=0$ is the only touchdown point provided that $f^{\prime}(r) \leq 0$. Most of the above results, existing in [9] for $p=2$, could be easily extended to the case $p>1$.

\section{Proof of Theorem 1.2}

We study the structure of the set of radially symmetric stationary solutions in the case of (1.3). This problem is reduced to

$$
\begin{aligned}
& \left(r^{n-1} u_{r}\right)_{r}+\frac{\lambda r^{n+\beta-1}}{(1-u)^{p}}=0, \quad 0 \leq u<1, \quad \text { for } r \in(0,1) \\
& u(1)=0, \quad u_{r}(0)=0
\end{aligned}
$$

where $u=u(r)$ for $r=|x|$.

We apply the phase-plane analysis $[13,17,18,19]$. First, putting $r=y^{\frac{2}{\beta+2}}$, we obtain

$$
\begin{aligned}
& \left(y^{a} u_{y}\right)_{y}+\frac{4 \lambda}{(\beta+2)^{2}} \frac{y^{a}}{(1-u)^{p}}=0, \quad 0 \leq u<1 \quad \text { for } y \in(0,1) \\
& u(1)=0, \quad \lim _{y \rightarrow 0} y^{\frac{\beta}{\beta+2}} u_{y}=0
\end{aligned}
$$

for $a=\frac{2 n+\beta-2}{\beta+2}$. 
We note that every positive solution of problem (3.2) can be obtained as a solution of the following initial value problem with certain positive constant $A$ :

$$
\begin{aligned}
& \left(y^{a} u_{y}\right)_{y}+\frac{4 \lambda}{(\beta+2)^{2}} \frac{y^{a}}{(1-u)^{p}}=0, \quad 0 \leq u<1, \quad \text { for } y \in(0,1) \\
& u(0)=A \in(0,1), \quad \lim _{y \rightarrow 0} y^{\frac{\beta}{\beta+2}} u_{y}=0 .
\end{aligned}
$$

Putting

$$
k=\sqrt{\frac{\{\beta+2+(n-2)(p+1)\}(\beta+2)(1-A)^{p+1}}{\lambda(p+1)^{2}}},
$$

we apply the Emden transformation

$$
u(y)=1-(1-A) e^{\frac{2}{p+1} t} w(t), \quad y=k e^{t} .
$$

Then, (3.3) is reduced to

$$
\begin{aligned}
& \ddot{w}+\frac{2(n p+n-2 p+2 \beta+2)}{(\beta+2)(p+1)} \dot{w}+\frac{4(n p+n-2 p+\beta)}{(\beta+2)(p+1)^{2}}\left(w-\frac{1}{w^{p}}\right)=0 \\
& 0<w<\frac{1}{1-A} e^{-\frac{2}{p+1} t} \quad \text { for } t \in \mathbb{R} \\
& \lim _{t \rightarrow-\infty} e^{\frac{2}{p+1} t} w=1, \quad \lim _{t \rightarrow-\infty} e^{\frac{2}{p+1} t} \dot{w}=-\frac{2}{p+1}
\end{aligned}
$$

The profile of this orbit is studied in detail in [13]. First, we show the following lemma.

Lemma 3.1. Problem (3.4) admits a unique solution for $t \ll-1$.

Proof. Through the transformation,

$$
v(z)=e^{\frac{2}{p+1} t} w(t), \quad z=e^{t}
$$

(3.4) is reduced to

$$
\begin{aligned}
& \left(z^{b} v_{z}\right)_{z}-\frac{4(n p+n-2 p+\beta)}{(p+1)^{2}(\beta+2)} z^{b} v^{-p}=0 \\
& 0<v<\frac{1}{1-A}, \quad z>0, \\
& v(0)=1, \quad \lim _{z \downarrow 0} z v_{z}=0,
\end{aligned}
$$

where $b=\frac{p \beta+\beta+2 n p+2 n-2 p-2}{(p+1)(\beta+2)}$ and then we obtain the integral equation

$$
v(z)=1+\frac{4(n p+n-2 p+\beta)}{(p+1)^{2}(\beta+2)} \cdot \int_{0}^{z} \sigma^{-b} d \sigma \int_{0}^{\sigma} \zeta^{b} v(\zeta)^{-p} d \zeta .
$$


Now, we consider the Banach space

$$
X=\{v=v(z) \mid v \in C([0, \varepsilon]) \text { and } v(0)=1\},
$$

equipped with the supremum norm $\|\cdot\|_{\infty}$, where $\varepsilon>0$ is a constant to be determined later. Let $\Psi(v) \in X$ be the right-hand side of (3.5) defined for $v \in X$. For, given

$$
v_{i} \in Y \equiv\left\{v \in X \mid 1 \leq v \leq \frac{1}{1-A}\right\}, \quad(i=1,2)
$$

we have

$$
\begin{aligned}
\left|\Psi\left(v_{1}\right)-\Psi\left(v_{2}\right)\right| & \leq \frac{4(n p+n-2 p+\beta)}{(p+1)^{2}(\beta+2)} \int_{0}^{z} \sigma^{-b} d \sigma \int_{0}^{\sigma} \zeta^{b}\left|v_{1}(\zeta)^{-p}-v_{2}(\zeta)^{-p}\right| d \zeta \\
& \leq \frac{4(n p+n-2 p+\beta)}{(p+1)^{2}(\beta+2)} \int_{0}^{z} \sigma^{-b} d \sigma \\
& \leq \frac{4 p(n p+n-2 p+\beta)}{(p+1)^{2}(\beta+2)(1-A)^{p-1}} \int_{0}^{\sigma} \sigma^{-b} d \sigma \int_{0}^{\sigma} \zeta^{b}\left\|v_{1}-v_{2}\right\|_{\infty} d \zeta \\
& \left.\leq \frac{4 p(n p+n-2 p+\beta)}{(p+1)^{2}(\beta+2)(1-A)^{p-1}} \int_{0}^{\varepsilon} \sigma d \sigma \| v_{1}(\zeta)+\left.(1-\theta) v_{2}(\zeta)\right|^{p-1} d \theta\right) \mid v_{1}(\zeta)-v_{2}(\zeta) \|_{\infty} v_{2}(\zeta)^{p} d \zeta \\
& \leq \frac{2 p(n p+n-2 p+\beta)}{(p+1)^{2}(\beta+2)(1-A)^{p-1}} \varepsilon^{2}\left\|v_{1}-v_{2}\right\|_{\infty} \cdot
\end{aligned}
$$

Therefore

$$
\left\|\Psi\left(v_{1}\right)-\Psi\left(v_{2}\right)\right\|_{\infty} \leq \frac{2 p(n p+n-2 p+\beta)}{(p+1)^{2}(\beta+2)(1-A)^{p-1}} \varepsilon^{2}\left\|v_{1}-v_{2}\right\|_{\infty} .
$$

Similarly, we obtain

$$
\begin{aligned}
|\Psi(v)| & \leq 1+\frac{4(n p+n-2 p+\beta)}{(p+1)^{2}(\beta+2)} \cdot \int_{0}^{z} \sigma^{-b} d \sigma \int_{0}^{\sigma} \zeta^{b}|v(\zeta)|^{-p} d \zeta \\
& \leq 1+\frac{2(n p+n-2 p+\beta)}{(p+1)^{2}(\beta+2)} \varepsilon^{2},
\end{aligned}
$$


and hence

$$
\|\Psi(v)\|_{\infty} \leq 1+\frac{2(n p+n-2 p+\beta)}{(p+1)^{2}(\beta+2)} \varepsilon^{2} .
$$

For

$$
\varepsilon \leq \min \left(\sqrt{\frac{(p+1)^{2}(\beta+2)(1-A)^{p-1}}{4 p(n p+n-2 p+\beta)}}, \sqrt{\frac{(p+1)^{2}(\beta+2) A}{2(n p+n-2 p+\beta)(1-A)}}\right)
$$

it holds that $\Psi\left(v_{i}\right) \in Y$ for $i=1,2$ and

$$
\left\|\Psi\left(v_{1}\right)-\Psi\left(v_{2}\right)\right\|_{\infty} \leq \frac{1}{2}\left\|v_{1}-v_{2}\right\|_{\infty} .
$$

Hence $\Psi$ has a unique fixed point $v \in X$ by the contraction mapping principle, and the proof is complete.

Now we write (3.4) as an autonomous system

$$
\frac{d}{d t}\left(\begin{array}{c}
w \\
\dot{w}
\end{array}\right)=\left(\begin{array}{c}
\dot{w} \\
-\frac{2(n p+n-2 p+2 \beta+2)}{(p+1)(\beta+2)} \dot{w}-\frac{4(n p+n-2 p+\beta)}{(p+1)^{2}(\beta+2)}\left(w-\frac{1}{w^{p}}\right)
\end{array}\right) .
$$

Note that $(w, \dot{w})=(1,0)$ is a singular-fixed point of this system. The linearized equation around this fixed point is given by

$$
\frac{d}{d t}\left(\begin{array}{l}
W \\
\dot{W}
\end{array}\right)=\left(\begin{array}{cc}
0 & 1 \\
-\frac{4(n p+n-2 p+\beta)}{(p+1)(\beta+2)} & -\frac{2(n p+n-2 p+2 \beta+2)}{(p+1)(\beta+2)}
\end{array}\right)\left(\begin{array}{l}
W \\
\dot{W}
\end{array}\right),
$$

and the eigenvalues $\mu_{ \pm}$of the coefficient matrix

$$
\left(\begin{array}{cc}
0 & 1 \\
-\frac{4(n p+n-2 p+\beta)}{(p+1)(\beta+2)} & -\frac{2(n p+n-2 p+2 \beta+2)}{(p+1)(\beta+2)}
\end{array}\right),
$$

are given as

$$
\mu_{ \pm}=\frac{-(n p+n-2 p+2 \beta+2) \pm \sqrt{f(p, n, \beta)}}{(p+1)(\beta+2)}
$$

where

$$
\begin{aligned}
f(p, n, \beta)= & (p+1)^{2} n^{2}-4(p+1)(\beta p+3 p+1) n \\
& -4\left\{\beta^{2} p+2 p \beta(1-p)-\left(5 p^{2}+2 p+1\right)\right\} .
\end{aligned}
$$

Since $n \geq 2$, we obtain $n p+n-2 p+2 \beta+2>0$. Thus, $(w, \dot{w})=(1,0)$ is an attractor. 
The curve $\dot{w}=\varphi(w)$ defined for

$$
\begin{aligned}
\varphi(w) & =-a\left(w-\frac{1}{w^{p}}\right) \\
a & =\frac{(p+1)(\beta+2)}{n p+n-2 p+2 \beta+2} \cdot \frac{2(n p+n-2 p+\beta)}{(p+1)^{2}(\beta+2)}>0
\end{aligned}
$$

passes through $(1,0)$ and the right-half plane $\{(w, \dot{w}) \mid w>0\}$ of the phase space is separated into four regions:

$$
\begin{aligned}
I & =\{(w, \dot{w}) \mid w>0, \dot{w}>0, \dot{w}>\varphi(w)\}, \\
I I & =\{(w, \dot{w}) \mid w>0, \dot{w}<0, \dot{w}>\varphi(w)\}, \\
I I I & =\{(w, \dot{w}) \mid w>0, \dot{w}<0, \dot{w}<\varphi(w)\}, \\
I V & =\{(w, \dot{w}) \mid w>0, \dot{w}>0, \dot{w}<\varphi(w)\} .
\end{aligned}
$$

According to these cases, the directions of the orbits $\mathcal{O}$ are as follows:

$$
\begin{aligned}
(w, \dot{w}) \in \mathcal{O} \cap I & \Rightarrow \frac{d w}{d t}>0, \frac{d \dot{w}}{d t}<0, \\
(w, \dot{w}) \in \mathcal{O} \cap I I & \Rightarrow \frac{d w}{d t}<0, \frac{d \dot{w}}{d t}<0, \\
(w, \dot{w}) \in \mathcal{O} \cap I I I & \Rightarrow \frac{d w}{d t}<0, \frac{d \dot{w}}{d t}>0, \\
(w, \dot{w}) \in \mathcal{O} \cap I V & \Rightarrow \frac{d w}{d t}>0, \frac{d \dot{w}}{d t}>0 .
\end{aligned}
$$

If $(w, \dot{w}) \in \mathcal{O}$ approaches $w=0$, then $\frac{d \dot{w}}{d t} \rightarrow+\infty$, and therefore it enters region $I V$. Thus, $\mathcal{O}$ in the right-half plane does not touch $w=0$ in finite time. If $(w, \dot{w}) \in \mathcal{O} \cap I$ with $w \gg 1$, then it must eventually come into region $I I$ because $\frac{d w}{d t}=\dot{w}>0$ and $\frac{d \dot{w}}{d t}=\gamma(-\dot{w}+\varphi(w)) \sim \gamma(-\dot{w}-a w)<0$, where $\gamma=2(n p+n-$ $2 p+2 \beta+2) /(p+1)(\beta+2)$. From those considerations, we see that the orbit $\mathcal{O}$ is compact in the right-half space, and eventually approaches the singular point $(w, \dot{w})=(1,0)$ as $t \rightarrow+\infty$.

The following elementary lemma controls the (local) behaviour of the orbit $\mathcal{O}$ around the singular-fixed point $(w, \dot{w})=(1,0)$.

Lemma 3.2. We have the following.

1. If $n \in[2,6]$, then $f(p, n, \beta)<0$ for any $p>1$ and $\beta>0$.

2. If $n \in[7,9], f(p, n, \beta) \geq 0$ for $p \in(1, \bar{p}]$ and $\beta \in(0, \bar{\beta}]$, and $f(p, n, \beta)<0$ otherwise, i.e. for $p>\bar{p}$ and $\beta>0$ or for $p \in(1, \bar{p})$ and $\beta>\bar{\beta}$, whenever $\bar{\beta}>0$.

3. If $n \geq 10, f(p, n, \beta) \geq 0$ for $p>1$ and $\beta \in(0, \bar{\beta}]$, and $f(p, n, \beta)<0$ otherwise. 
Proof. We have

$$
f(p, n, \beta)=-4 p\left(\beta-\frac{2(p-1)-(p+1) n}{2}\right)^{2}+(p+1)^{3}(n-2)^{2}
$$

Since

$$
2(p-1)-(p+1) n=-(p+1)(n-2)-4<0,
$$

$f(p, n, \beta)$ achives the maximum at $\beta=0$ for fixed $p>1$ and $n \geq 2$ with the value

$$
\begin{aligned}
f(p, n, 0) & =(p+1)^{2} n^{2}-4(p+1)(3 p+1) n+4\left(5 p^{2}+2 p+1\right) \\
& =(n-2)(n-10) p^{2}+2\left(n^{2}-8 n+4\right) p+(n-2)^{2}
\end{aligned}
$$

By a simple computation, we have

$$
f(p, n, 0)<0 \quad \text { for } n=2, \cdots, 6 \text { and } p>1,
$$

and

$$
\begin{array}{ll}
f(p, n, 0) \geq 0 & \text { for } p \in(1, \bar{p}] \text { and } n=7,8,9 \\
f(p, n, 0)<0 & \text { for } p>\bar{p} \text { and } n=7,8,9 .
\end{array}
$$

Then, the first and second cases of the lemma are obtained by the definion of $\bar{\beta}$. Since

$$
f(1, n, 0)=4\left(n^{2}-8 n+8\right)
$$

and $f(p, n, 0)$ is increasing with respect to $p>0$ for $n \geq 10$, we obtain $f(p, n, \beta) \geq$ 0 for $p \geq 1, n \geq 10$, and $\beta \in(0, \bar{\beta}]$, while $f(p, n, \beta)<0$ for $n \geq 10, p>1$ and $\beta>\bar{\beta}$. This completes the proof of the lemma.

Lemma 3.2 guarantees that the singular point $(1,0)$ is a spiral attractor if and only if

$$
\begin{array}{ll}
n \in[2,6], & p>1, \beta>0,
\end{array}
$$

while in the secondary case, i.e. when

$$
\begin{aligned}
& n \in[7,9], \quad p \in(1, \bar{p}], \beta \in(0, \bar{\beta}], \\
& n \geq 10, \quad p>1, \beta \in(0, \bar{\beta}]
\end{aligned}
$$

the orbit $\mathcal{O}$ is absorbed into $(1,0)$ exponentially. 
Through the above transformation, the boundary condition $u(1)=0$ in $(3.1)$ corresponds to

$$
\begin{aligned}
w(\tau) & =\left\{\frac{[\beta+2+(n-2)(p+1)](\beta+2)}{\lambda(p+1)^{2}}\right\}^{\frac{1}{p+1}} \\
\tau & =-\frac{1}{2} \log \frac{\{\beta+2+(n-2)(p+1)\}(1-A)^{p+1}}{\lambda(p+1)^{2}}=\log \frac{1}{k} .
\end{aligned}
$$

In other words, for any $\tau \in \mathbb{R},\left(\lambda_{\tau}, u_{\tau}\right)$ defined by

$$
\begin{aligned}
u_{\tau}(r) & =1-\frac{w\left(\tau+\frac{\beta+2}{2} \log r\right)}{w(\tau)} r^{\frac{\beta+2}{p+1}} \\
\lambda_{\tau} & =\frac{\{\beta+2+(n-2)(p+1)\}(\beta+2)}{(p+1)^{2} w(\tau)^{p+1}} \\
A_{\tau} & =1-\frac{1}{e^{\frac{2}{p+1} \tau} w(\tau)}
\end{aligned}
$$

satisfies (3.1), and conversely, every solution of (3.1) is written in the form of (3.6). Hence $\mathcal{C}_{r}$ is homeomorphic to $\mathcal{O}$. Since the singular point $(w, \dot{w})=(1,0)$ corresponds to $(\lambda, u)=\left(\lambda^{*}, 1-|x|^{\frac{\beta+2}{p+1}}\right)$, we obtain the conclusion of Theorem 1.2.

\section{Proof of Theorem 1.3}

Theorem 1.3 is proven by the method of [19]. Each $(\lambda, u) \in \mathcal{C}_{r}$ corresponds to $(w(\tau), \dot{w}(\tau)) \in \mathcal{O}$ and therefore, it is parametrized by $\tau \in \mathbb{R}:(\lambda, u)=(\lambda(\tau), u(\tau))$. We denote by $\mu_{\tau}^{\ell}, \ell=1,2, \ldots$, the $\ell$-th eigenvalue of the linearized problem (1.4) corresponding to a radially symmetric eigenfuction, i.e. a function satisfying

$$
\begin{gathered}
-\phi_{r r}-\frac{n-1}{r} \phi_{r}-\frac{p \lambda r^{\beta}}{(1-u)^{p+1}} \phi=\mu \phi \quad(0<r<1) \\
\phi_{r}(0)=\phi(1)=0 .
\end{gathered}
$$

Each $\mu_{\tau}^{\ell}$ is simple. If $(\lambda(\tau), u(\tau))$ is on the turning point of $\mathcal{C}_{r}$, then, by the implicit function theorem, there is $\ell \geq 1$ such that $\mu_{\tau}^{\ell}=0$. If $\mu_{\tau}^{\ell}=0$ holds for some $\ell \geq 1$ with $(\lambda(\tau), u(\tau)) \in \mathcal{C}_{r}$ not on the turning point, then, by the bifurcation theory on the critical point of odd multiplicity, [23, 24], it is actually the bifurcation point of $\mathcal{C}_{r}$. But, this is impossible by Theorem 1.2, and therefore, $(\lambda(\tau), u(\tau))$ is on the turning point of $\mathcal{C}_{r}$ if and only if (4.1) has the eigevalue 0. Denoting the turning points of $\mathcal{C}_{r}$ by $T_{k}=\left(\lambda\left(\tau_{k}\right), u\left(\tau_{k}\right)\right)\left(\tau_{1}<\tau_{2}<\cdots\right)$, we only have to show $\dot{\mu}_{\tau=\tau_{k}}^{\ell(k)}<0$ for $k \geq 1$ and $\ell=\ell(k)$ such that $\mu_{\tau_{k}}^{\ell(k)}=0$, to prove Theorem 1.3. 
Using (3.6), we have

$$
\begin{aligned}
& \dot{\lambda}(\tau)=-\frac{\{\beta+2+(n-2)(p+1)\}(\beta+2)}{p+1} \frac{\dot{w}(\tau)}{w(\tau)^{p+2}} \\
& \ddot{\lambda}(\tau)=-\frac{\{\beta+2+(n-2)(p+1)\}(\beta+2)}{p+1} \frac{\ddot{w}(\tau) w(\tau)-(p+2) \dot{w}(\tau)^{2}}{w(\tau)^{p+3}},
\end{aligned}
$$

where here and above ${ }^{\circ}=\frac{d}{d \tau}$, stands for the differentiation with respect to $\tau$. On the other hand, from (1.1) it holds that

$$
\begin{aligned}
& \dot{u}_{r r}+\frac{n-1}{r} \dot{u}_{r}+\frac{\dot{\lambda} r^{\beta}}{(1-u)^{p}}+\frac{p \lambda r^{\beta}}{(1-u)^{p+1}} \dot{u}=0, \quad(0<r<1) \\
& \dot{u}_{r}(0)=\dot{u}(1)=0 .
\end{aligned}
$$

Due to (3.6) we have

$$
\dot{u}(r, \tau)=\frac{\dot{w}(\tau) w\left(\tau+\frac{\beta+2}{2} \log r\right)-\dot{w}\left(\tau+\frac{\beta+2}{2} \log r\right) w(\tau)}{w(\tau)^{2}} r^{\frac{\beta+2}{p+1}}
$$

and since $\mathcal{O}$ does not contain a segment we derive that $\dot{u}(r, \tau) \not \equiv 0$. Moreover, since $\dot{\lambda}\left(\tau_{k}\right)=0$ in (4.3), we can take $\phi\left(\cdot, \tau_{k}\right)=\dot{u}\left(\cdot, \tau_{k}\right)$. Thus, the perturbation theory, see [14], guarantees the existence of $\phi=\phi(\cdot, \tau)$ and $\mu=\mu(\tau)$ smooth in $\tau$ for $\left|\tau-\tau_{k}\right| \ll 1$ satisfying $(4.1), \phi\left(\cdot, \tau_{k}\right)=\dot{u}\left(\cdot, \tau_{k}\right)$, and $\mu\left(\tau_{k}\right)=\mu_{\tau_{k}}^{\ell(k)}=0$ since $\mu_{\tau_{k}}^{\ell(k)}$ is simple.

Differentiating (4.3) and (4.1) with respect to $\tau$, we have

$$
\begin{aligned}
\Delta_{r} \ddot{u} & +\frac{\ddot{\lambda} r^{\beta}}{(1-u)^{p}}+\frac{p \dot{\lambda} r^{\beta}}{(1-u)^{p+1}} \dot{u}+\frac{p \dot{\lambda} r^{\beta}}{(1-u)^{p+1}} \dot{u}+\frac{p(p+1) \lambda r^{\beta}}{(1-u)^{p+2}} \dot{u}^{2} \\
& +\frac{p \lambda r^{\beta}}{(1-u)^{p+1}} \ddot{u}=0 \quad \text { in } \Omega, \quad \ddot{u}=0 \quad \text { on } \partial \Omega,
\end{aligned}
$$

and

$$
\begin{aligned}
& -\Delta_{r} \dot{\phi}-\frac{p \dot{\lambda} r^{\beta}}{(1-u)^{p+1}} \phi-\frac{p(p+1) \lambda r^{\beta}}{(1-u)^{p+2}} \dot{u} \phi-\frac{p \lambda r^{\beta}}{(1-u)^{p+1}} \dot{\phi} \\
& =\dot{\mu} \phi+\mu \dot{\phi} \quad \text { in } \Omega, \quad \dot{\phi}=0 \quad \text { on } \partial \Omega,
\end{aligned}
$$

where $\Delta_{r}=\partial^{2} / \partial r^{2}+\frac{n-1}{r} \partial / \partial r$.

Multiplying (4.4) and (4.5) by $\dot{u}$, integrating over $\Omega$, and subtracting each other, we obtain

$$
\ddot{\lambda}\left(\tau_{k}\right) \int_{\Omega} \frac{r^{\beta}}{\left(1-u_{k}\right)^{p}} \phi\left(\tau_{k}\right) d x=\dot{\mu}\left(\tau_{k}\right) \int_{\Omega} \phi\left(\tau_{k}\right)^{2} d x
$$

in the bending point $\tau=\tau_{k}$, where $u_{k}=u\left(\cdot, \tau_{k}\right)$. 
Let

$$
I_{k}=\int_{\Omega} \frac{r^{\beta}}{\left(1-u_{k}\right)^{p}} \phi\left(\tau_{k}\right) d x .
$$

The following lemma implies

$$
\begin{aligned}
& \dot{\mu}\left(\tau_{k}\right) \int_{\Omega} \phi\left(\tau_{k}\right)^{2} d x=\ddot{\lambda}\left(\tau_{k}\right) I_{k} \\
& \quad=-\frac{\{\beta+2+(n-2)(p+1)\}(\beta+2)^{2} \omega_{n}}{2(p+1)^{2} \lambda_{k}} \frac{\ddot{w}\left(\tau_{k}\right)^{2}}{w\left(\tau_{k}\right)^{p+3}}
\end{aligned}
$$

by $(4.2)$, where $\omega_{n}$ denotes the area of the unit sphere in $\mathbb{R}^{n}$, and hence $\dot{\mu}\left(\tau_{k}\right)<0$.

Lemma 4.1. It holds that

$$
I_{k}=\frac{(\beta+2) \omega_{n}}{2(p+1) \lambda_{k}} \frac{\ddot{w}\left(\tau_{k}\right)}{w\left(\tau_{k}\right)}
$$

where $\lambda_{k}=\lambda\left(\tau_{k}\right)$.

Proof. We have

$$
\begin{aligned}
\lambda(p-1) \int_{\Omega} \frac{r^{\beta}}{(1-u)^{p}} \dot{u} d x+\dot{\lambda} \int_{\Omega} \frac{r^{\beta}}{(1-u)^{p-1}} d x=\frac{d}{d \tau}\left(\int_{\Omega} \frac{\lambda r^{\beta}}{(1-u)^{p-1}} d x\right) \\
=\frac{d}{d \tau} \int_{\Omega}\left(\Delta_{r} u\right)(u-1) d x=-\int_{\Omega} \Delta_{r} \dot{u} d x+\frac{d}{d \tau} \int_{\Omega} u \Delta_{r} u d x \\
=-\int_{\Omega} \Delta_{r} \dot{u} d x-\frac{d}{d \tau} \lambda \int_{\Omega} \frac{r^{\beta}}{(1-u)^{p}} u d x=-\int_{\Omega} \Delta_{r} \dot{u} d x-\dot{\lambda} \int_{\Omega} \frac{r^{\beta}}{(1-u)^{p}} u d x \\
\quad-\lambda \int_{\Omega} \frac{r^{\beta}}{(1-u)^{p}} \dot{u} d x-p \lambda \int_{\Omega} \frac{r^{\beta}}{(1-u)^{p+1}} u \dot{u} d x
\end{aligned}
$$

and therefore,

$$
(p-1) \lambda_{k} I_{k}=-\int_{\Omega} \Delta_{r} \dot{u}_{k} d x-\lambda_{k} I_{k}-p \lambda_{k} \int_{\Omega} \frac{r^{\beta}}{\left(1-u_{k}\right)^{p+1}} u_{k} \dot{u}_{k} d x,
$$

by putting $\tau=\tau_{k}$, where $\dot{u}_{k}=\dot{u}\left(\cdot, \tau_{k}\right)$. This means

$$
p \lambda_{k} I_{k}=-\int_{\Omega} \Delta_{r} \dot{u}_{k} d x-p \lambda_{k} \int_{\Omega} \frac{r^{\beta}}{\left(1-u_{k}\right)^{p+1}} u_{k} \dot{u}_{k} d x
$$


Since $\dot{u}_{k}=\dot{u}\left(r, \tau_{k}\right)$, we have

$$
\int_{\Omega} \Delta_{r} \dot{u}_{k} d x=\omega_{n}\left(\dot{u}_{k}\right)_{r}(1),
$$

while

$$
u(r, \tau)=1-\frac{w\left(\tau+\frac{\beta+2}{2} \log r\right)}{w(\tau)} r^{\frac{\beta+2}{p+1}}
$$

implies

$$
\begin{aligned}
\dot{u}_{r}(r, \tau)= & -\frac{\beta+2}{p+1} \frac{w(\tau) \dot{w}\left(\tau+\frac{\beta+2}{2} \log r\right)-\dot{w}(\tau) w\left(\tau+\frac{\beta+2}{2} \log r\right)}{w(\tau)^{2}} r^{\frac{\beta+2}{p+1}-1} \\
& -\frac{\beta+2}{2} \frac{w(\tau) \ddot{w}\left(\tau+\frac{\beta+2}{2} \log r\right)-\dot{w}(\tau) \dot{w}\left(\tau+\frac{\beta+2}{2} \log r\right)}{w(\tau)^{2}} r^{\frac{\beta+2}{p+1}-1} .
\end{aligned}
$$

Here, we have $\dot{w}\left(\tau_{k}\right)=0$ by (4.2) and $\dot{\lambda}\left(\tau_{k}\right)=0$ and hence

$$
\int_{\Omega} \Delta_{r} \dot{u}_{k} d x=-\frac{(\beta+2) \omega_{n}}{2} \frac{\ddot{w}\left(\tau_{k}\right)}{w\left(\tau_{k}\right)} .
$$

Multiplying (4.1) by $u$ and integrating over $\Omega$, we obtain

$$
-\int_{\Omega} u \Delta_{r} \phi d x-\int_{\Omega} \frac{p \lambda r^{\beta}}{(1-u)^{p+1}} \phi u d x=\mu \int_{\Omega} \phi u d x
$$

and hence

$$
\begin{aligned}
\int_{\Omega} \frac{p \lambda r^{\beta}}{(1-u)^{p+1}} \phi u d x & =-\mu \int_{\Omega} \phi u d x-\int_{\Omega} \Delta_{r} u \phi d x \\
& =-\mu \int_{\Omega} \phi u d x+\lambda \int_{\Omega} \frac{r^{\beta}}{(1-u)^{p}} \phi d x
\end{aligned}
$$

Putting $\tau=\tau_{k}$, we have

$$
\int_{\Omega} \frac{p \lambda_{k} r^{\beta}}{\left(1-u_{k}\right)^{p+1}} \dot{u}_{k} u_{k} d x=\lambda_{k} I_{k}
$$

Hence substituting (4.7) and (4.8) into (4.6), we finally derive

$$
I_{k}=\frac{(\beta+2) \omega_{n}}{2(p+1) \lambda_{k}} \frac{\ddot{w}\left(\tau_{k}\right)}{w\left(\tau_{k}\right)}
$$

and the proof is complete. 


\section{Connecting orbits}

Problem (1.1) generates a local semi-flow in $X=W_{0}^{1, q}(\Omega)$ for $q>n$, and each $\varphi \in \mathcal{C}^{\lambda}$ is regarded as an equilibrium. It is said to be hyperbolic if (1.4) does not admit $\mu=0$ as an eigenvalue. Given $\varphi, \psi \in \mathcal{C}^{\lambda}$, we define the stable manifold $W^{s}(\varphi)$ of $\varphi$ and the unstable manifold $W^{u}(\psi)$ of $\psi$ by

$$
\begin{aligned}
& W^{s}(\phi)=\{\xi \in X \mid \text { there exists an orbit } u:[0,+\infty) \rightarrow X \text { to }(1.1) \\
& \text { such that } u(0)=\xi \text { and } u(t) \rightarrow \phi \text { in } X \text { as } t \rightarrow+\infty\}, \\
& W^{u}(\psi)=\{\xi \in X \mid \text { there exists an orbit } u:(-\infty, 0] \rightarrow X \text { to }(1.1) \\
& \text { such that } u(0)=\xi \text { and } u(t) \rightarrow \psi \text { in } X \text { as } t \rightarrow-\infty\} .
\end{aligned}
$$

If there is a solution $u=u(\cdot, t)$ to (1.1) for $t \in \mathbb{R}$ satisfying $\lim _{t \rightarrow-\infty} u(t)=\psi$ and $\lim _{t \rightarrow+\infty} u(t)=\varphi$ in $X$, we call $\{u(\cdot, t) \mid t \in \mathbb{R}\}$ a connecting orbit between $\varphi$ and $\psi$.

In the case of (1.3), we can define radially symmetric versions of the above notions. Here, we just note that $\varphi \in \mathcal{C}_{r}^{\lambda}$ is called radially hyperbolic if (4.1) does not admit $\mu=0$ as an eigenvalue, and that radial stable and unstable manifolds of $\varphi \in \mathcal{C}_{r}^{\lambda}$ are denoted by $W_{r}^{s}(\varphi)$ and $W_{r}^{u}(\psi)$, respectively. Then, we obtain the Morse-Smale property of radially symmetric orbits [1, 6, 22].

Theorem 5.1. Let $\varphi, \psi \in \mathcal{C}_{r}^{\lambda}$ be radially hyperbolic and assume the existence of a radial connecting orbit $\{u(\cdot, t) \mid t \in \mathbb{R}\}$ between $\varphi$ and $\psi$. Then $W_{r}^{s}(\varphi)$ and $W_{r}^{u}(\psi)$ intersect transversally in $X_{r}=\{v \in X \mid v=v(|x|)\}$, and

$$
T_{u_{0}} W_{r}^{s}(\varphi)+T_{u_{0}} W_{r}^{u}(\psi)=X_{r}
$$

for $u_{0}=u(\cdot, 0)$, where $T_{u_{0}} W$ denotes the tangential space of $W$ at $u_{0}$. It also holds that $i_{R}(\lambda, \varphi)<i_{R}(\lambda, \psi)$.

To confirm this, we denote by $\mu_{k}$ the $k$-th eigenvalue of the linearized problem (4.1) around $\psi \in \mathcal{C}_{r}^{\lambda}$. For given $0<k<i_{R}(\lambda, \psi)$ and $\mu_{k}<\mu<\min \left\{0, \mu_{k+1}\right\}$, we consider a finer invariant manifold than the unstable manifold as

$$
\begin{aligned}
& W_{r, k}^{u}(\psi)=\left\{u_{0} \in W_{r}^{u}(\psi) \mid \lim _{t \rightarrow-\infty} e^{-\mu t}\|u(\cdot, t)-\psi\|_{X_{r}}=0,\right. \\
& \left.\quad \text { where } u(\cdot, t)(t \leq 0) \text { is a solution to (1.1) satisfying } u(\cdot, 0)=u_{0}\right\} .
\end{aligned}
$$

Theorem 5.1 is obtained by the discrete Lyapunov function. To state this, for given $\Psi \in C[0,1]$, we denote by $\ell(\Psi)$ the supremum of $k$ such that there is $0 \leq r_{1}<r_{2}<\cdots<r_{k} \leq 1$ satisfying $\Psi\left(r_{j}\right) \Psi\left(r_{j+1}\right)<0$ for $j=1,2, \cdots, k$. Then, Lemma 2.9 of [6] is stated as follows.

Lemma 5.2. Given radially hyperbolic $\varphi, \psi \in \mathcal{C}_{r}^{\lambda}$, we obtain the following. 
1. If $u_{0}, u_{1} \in W_{r, k}^{u}(\psi), w \in T_{u_{0}} W_{r, k}^{u}(\psi), u_{0} \neq u_{1}$, and $w \neq 0$, then it holds that $\ell\left(u_{0}-u_{1}\right) \leq k$ and $\ell(w) \leq k$.

2. If $u_{0}, u_{1} \in W_{r}^{s}(\varphi), w \in T_{u_{0}} W_{r}^{s}(\varphi), u_{0} \neq u_{1}$, and $w \neq 0$, then it holds that $\ell\left(u_{0}-u_{1}\right) \geq i_{R}(\lambda, \varphi)+1$ and $\ell(w) \geq i_{R}(\lambda, \varphi)+1$.

Proof of Theoerem 5.1. The proof is similar to that of Lemma 3.1 in [6]. First, we note

$$
u_{0} \in W_{r}^{s}(\varphi) \cap W_{r}^{u}(\psi) \neq \emptyset
$$

and hence $i_{R}(\lambda, \varphi)<i_{R}(\lambda, \psi)$ by Lemma 5.2. This implies

$$
W_{r, i_{R}(\lambda, \varphi)}^{u}(\psi) \subset W_{r, i_{R}(\lambda, \psi)}^{u}(\psi),
$$

where the dimension of $T_{u_{0}} W_{r}^{u}(\varphi)$ and the codimension of $T_{u_{0}} W_{r}^{s}(\varphi)$ are equal to $i_{R}(\lambda, \varphi)$. Therefore it is sufficient to show

$$
T_{u_{0}} W_{r, i_{R}(\lambda, \varphi)}^{u}(\psi) \cap T_{u_{0}} W_{r}^{s}(\varphi)=\{0\},
$$

but this is an immediate consequence of Lemma 5.2, hence

$$
T_{u_{0}} W_{r}^{s}(\varphi)+T_{u_{0}} W_{r}^{u}(\psi)=X_{r} .
$$

\section{Acknowledgement}

N.I. Kavallaris was supported by the 21st Century Center of Excellence Program "Towards a New Basic Science : Depth and Synthesis". He would like also to thank the Division of Mathematical Science of Graduate School of Engineers Science of Osaka University of its hospitality during the preparation of this manuscript.

\section{References}

[1] S. B. Angenent, The Morse-Smale property for a semi-linear parabolic equation. J. Differential Equations. 62 (1986), 427-442.

[2] H. Brezis, T. Cazenave, Y. Martel, and A. Ramiandrisoa, Blow up for $u_{t}-$ $\Delta u=g(u)$ revisited. Adv. Differential Equations 1 (1996), 73-90.

[3] K-S Chou and G-F Zhenh, Some dichotomy results for the quenching problem, preprint.

[4] P. Esposito, N. Ghoussoub and Y. Guo, Compactness along the first branch of unstable solutions for an elliptic problem with a singular nonlinearity. Comm. Pure Appl. Math. 60 (2007), 1731-1768.

[5] A. Friedman and B. McLeod, Blow-up of positive solutions of semilinear heat equations. Indiana Uni. Math. J. 34 (1985), 425-447. 
[6] M. Fila, H. Matano and P. Poláčik, Existence of $L^{1}$-connection between equilibria of a semilinear parabolic equation. J. Dynamics and Differential Equations. 14 (2002), 463-491.

[7] H. Fujita, On the nonlinear equations $\Delta u+e^{u}=0$ and $\partial v / \partial t=\Delta v+e^{v}$. Bull. Amer. Math. Soc. 75 (1969), 132-135.

[8] N. Ghoussoub and Y. Guo, On the partial differential equations of electrostatic MEMS devices: stationary case. SIAM J. Math. Anal. 38 (2007), 14231449 .

[9] N. Ghoussoub and Y. Guo, On the partial differential equations of electrostatic MEMS devices II: dynamic case. Nonlinear Diff. Eqns. Appl. in press.

[10] B. Gidas, W.-M. Ni and L. Niereberg, Symmetry and related properties via the maximum principle. Comm. Math. Phys. 68 (1979), 209-243.

[11] Y. Guo, Z. Pan and M. J. Ward, Touchdown and pull-in voltage behavior of a MEMS device with varying dielectric properties, SIAM J. Appl. Math. 166 (2006), 309-338.

[12] Y. Guo, On the partial differential equations of electrostatic MEMS devices III:Refined Touchdown Behaviour, submitted in J. Differential Equations.

[13] D. D. Joseph and T. S. Lundgren, Quasilinear Dirichlet problems driven by positive sources. Arch. Rational Mech. Anal. 49 (1973), 241-269.

[14] T. Kato, Perturbation Theory for Linear Operators, Springer, Berlin, 1966.

[15] A. Kohda and T. Suzuki, Blow-up criteria for semilinear parabolic equations. J. Math. Anal. Appl. 243 (2000), 127-139.

[16] C.-S. Lin and W.-M. Ni, A counterexample to the nodal domain conjecture and a related semilinear equation. Proc. Am. Math. Soc. 102 (1988), 271-277.

[17] T. Miyasita and T. Suzuki, Non-local Gel'fand problem in higher dimension, Nonlocal Elliptic and Parabolic Problems, Banach Center Publ. 66 (2004), $221-235$.

[18] K. Nagasaki and T. Suzuki, Radial solutions for $\Delta u+\lambda e^{u}=0$ on annuli in higher dimensions. J. Differential Equations. 100 (1992), 137-161.

[19] K. Nagasaki and T. Suzuki, Spectral and related properties about the EmdenFowler equation $-\Delta u=\lambda e^{u}$ on circular domains. Math. Ann. 299 (1994), $1-15$.

[20] Y. Naito and T. Suzuki, Radial symmetry of positive solutions for semilinear elliptic equations on the unit ball in $\mathbb{R}^{n}$. Funkcial Ekvac. 41 (1998), 215-234.

[21] J. A. Pelesko and D. H. Bernstein, Modeling MEMS and NEMS, Chapman Hall and CRC Press, 2002. 
[22] P. Poláčik, Transversal and nontransversal intersections of stable and unstable manifolds in reaction diffusion equations on symmetric domains. Differential and Integral Equations. 7 (1994), 1527-1545.

[23] P. H. Rabinowitz, Some global results for nonlinear eigenvalue problems. J. Func. Anal. 7 (1971), 487-513.

[24] P. H. Rabinowitz, Some aspects of nonlinear eigenvalue problems, Rocky Mountain J. Math. 3 (1973), 161-202.

Nikos I. Kavallaris

Division of Mathematical Science

Graduate School of Engineering Science

Osaka University

Toyonaka 560-8531

Japan

email: nkaval@sigmath.es.osaka-u.ac.jp

Tosiya Miyasita

Department of Mathematics

Graduate School of Science

Kyoto University

Kyoto 606-8502

Japan

email: miyasita@math.kyoto-u.ac.jp

Takashi Suzuki Division of Mathematical Science

Graduate School of Engineering Science

Osaka University

Toyonaka 560-8531

Japan

email: suzuki@sigmath.es.osaka-u.ac.jp

Received: 22 May 2007

Accepted: 7 January 2008

Published Online First 03 October 2008

To access this journal online: http://www.birkhauser.ch 
